\title{
Comparison of Postoperative Fever and Effectiveness of Percutaneous Nephrolithotomy (PCNL) Versus Retrograde Intrarenal Surgery (RIRS) for the Treatment of Renal Stones
}

\author{
N. P. Gill ${ }^{1,2} \cdot$ L. D'Arrigo ${ }^{3}$ - E. C. Wit ${ }^{1,4}$ (I)
}

Accepted: 12 November 2018 / Published online: 20 December 2018

(C) The Author(s) 2018

\begin{abstract}
The aim of this study is to compare the effectiveness and the relative risk of postoperative fever between percutaneous nephrolithotomy and retrograde intrarenal surgery for the treatment of renal stones. At the Cannizzaro Hospital in Catania, a total 168 patients with one or more renal stones underwent one of more interventions, resulting in 177 independent records; 62 records related to PCNL treatment and 115 records involved RIRS treatment. To assess the relationship between patient and stone characteristics on the one hand and stone-free rate and the incidence rate of postoperative fever on the other, a generalized additive logistic models was used. We found that stone burden, defined in terms of stone diameter, the number of stones and stone density, is a significant predictor for the success of the intervention. Furthermore, PCNL treatment was significantly less successful for stone in the middle calyx, whereas RIRS obtained significantly lower success rate for stone in the lower calyx that had a sharp infundibular angle. Importantly, for renal stones with a diameter up to $2 \mathrm{~cm}$, RIRS has a very similar success rate compared to PCNL. Stones with a diameter larger than $2 \mathrm{~cm}$ are best treated by PCNL. Across the two treatments, older patients and men have lower incidence rate of postoperative fevers. The fact that RIRS has much lower postoperative fever rates for male and younger patients compared to PCNL, means that RIRS could be the treatment of choice for small and midsized stones for such patients.
\end{abstract}

Keywords Renal calculi $\cdot$ Stone-free $\cdot$ Sepsis $\cdot$ Flexible ureteroscopy $\cdot$ Percutaneous nephrolithotomy

\section{Introduction}

Percutaneous nephrolithotomy (PCNL) and retrograde intrarenal surgery (RIRS) are two common and widely used alternative interventions for the treatment of renal stones. Flexible

This article is part of the Topical Collection on Surgery

E. C. Wit

e.c.wit@rug.nl

1 Bernoulli Institute for Mathematics and Computer Science, University of Groningen, Groningen, The Netherlands

2 Department of Statistics, University of Sindh, Jamshoro, Pakistan

3 Urology Unit, Cannizzaro Hospital, Via Messina 329, Catania, Italy

4 Institute of Computational Science, Universita della Svizzera Italiana, 6900 Lugano, Switzerland ureteroscopy by RIRS is a popular intervention for the treatment of renal stones, and it has been widely used over the last two decades, particularly in Europe [7].The 2014 Urolithiasis Guideline of the European Association of Urology prescribes PCNL as the preferred treatment for renal stones over $2 \mathrm{~cm}$ in stone diameter [8], as it has high stone-free rates. However, it has been observed that PCNL in certain cases also lead to a high risk of postoperative fever, such as sepsis, which is a life-threatening and complicated infection.

Some studies have reported that RIRS is a successful treatment for larger-sized stones [3]. In a comparative study of 79 patients between PCNL and RIRS for renal stones with 15 to $20 \mathrm{~mm}$ stone diameter, the authors conclude that RIRS can be a good option for medium-sized stones and their reported stone-free rates in RIRS group were $89.2 \%$ after a single session and $94.6 \%$ after two sessions. Few comparative studies exits for the relative effectiveness of both treatments on larger renal stones. In a comparison study between PCNL and RIRS for 2-4 cm kidney stones [2], the authors conclude that performing multisession RIRS 
can achieve satisfactory results with low morbidity, and therefore, RIRS may be used as an alternative to PCNL. The stone-free rates they reported for RIRS and PCNL were 73.5 and $91.2 \%$, respectively.

The selection of the treatment usually depends on the patient and stone characteristics, in addition to patient personal preference. The use of RIRS has increased relative to percutaneous nephrolithotomy and shock wave lithotripsy (SWL) [4]. The comparison of PCNL or RIRS, in terms of high stone-free rates, should be complemented by a comparison of the treatments in terms of major complications, such as postoperative fever. Particularly, sepsis is a major complication and one of common causes of perioperative mortality [6]. To minimize the risk of sepsis, it is important to identify factors that increase risk in order to identify the patients that may be particularly susceptible to postoperative fever.

The aims of our study is to find the predictive parameters for stone-free and postoperative fever rates for both PCNL and RIRS treatments, and to make to recommendations which patients could benefit from these treatments.

\section{Material and Methods}

\section{Subjects Population}

In the present study a total of 168 patients were included, resulting in 177 medical records of single sessions, all of whom underwent treatment for renal stones over the period of March 2011 to December 2013. Among 177 medical records, 62 records involved a PCNL treatment and 115 in RIRS treatment. The patients had small to large renal stones (6-76 $\mathrm{mm}$ ) and sometimes multiple stones at multiple locations. All the patients were treated by two urologists at Cannizzaro Hospital, Catania, Italy. Both had experience of both interventions, treating more than 50 cases prior to this study.

\section{Basic Parameters}

We recorded basic information of various clinical parameters in the patient's records before undergoing to PCNL and RIRS treatment. Basic available information consists of age, gender, number of renal stones, stone location, stone diameter, stone volume, stone area, stone density (maximum and minimum), and operation time in minutes. Previous history of treatment and complications were also recorded. The stone size of stone diameter was measured using digital images of computer tomography (CT). The stone volume was calculated using the formula $\frac{1}{6} \pi \times$ length $\times$ width $\times$ height and formula used for stone area was $\frac{1}{3} \pi$ ( length $\times$ width + length $\times$ height + width $\times$ height). Renal stone density was measured in HU, the Hounsfield units. Blood and urine tests were performed in all patients. Patients who developed urinary tract infection were treated 5 days before undergoing to procedure with specific antibiotics. Three months after the treatments a patient was declared stone-free, when no renal stones were present or if the residual stone size was smaller than $4 \mathrm{~mm}$, when measured by postoperative ultrasound and KUB. Both treatments RIRS and PCNL were performed under anesthesia.

\section{Percutaneous Lithotripscy}

Before the PCNL procedure, a $6 \mathrm{~F}$ ureteral catheter is inserted through a cystoscope. The percutaneous access into the renal pelvis was performed by the urologist. Retrograde pyelography is conducted at the beginning of the procedure. The renal puncture was done under fluoroscopic and ultrasonography control. The telescopic dilation in classic prone position was used under fluoroscopic control through the calix. The patients underwent to endoscopic combined surgery (ECIRS) were excluded. The choice of calyx was related to stone location. When a supine procedure was performed, a pneumatic balloon for dilation was used. Finally, a $24 \mathrm{~F}$ Amplatz sheath was positioned, and an ultrasonic or pneumatic lithotripter used for lithotripsy. Nefroscope of $22 \mathrm{ch}$ (R. Wolf Company) with continuous flow irrigation was used. For the removal of the fragment, a tong or a basket (CooK company) was used, and at the end of PCNL, a disconnectable nephrostomy tube of 16 or 18 ch (Teleflex Company) was inserted into the renal pelvis. The operative time was evaluated from the puncture until the removal of the Amplatz sheath.

\section{Retrograde Intrarenal Surgery}

For the RIRS treatment, a flexible URS (Flex-X2,TM Storz, Germany) with holmium laser lithotripsy was used. For the stone pulverization, the energy level of a holmium YAG laser was set by the operator (urologist) based on his experience of dealing with urinary stone types. After the treatment, a urinary stent DJ was inserted into the ureter. This stent was removed within the 2 weeks after the procedure. When the operation time was longer more than the 90 mins, the RIRS procedure immediately was stopped and a DJ stent was inserted. In these patients, a second operation was performed within the next 3 weeks.

\section{Statistical Analysis}

Patients and stone characteristics were assessed and compared at baseline for PCNL and RIRS treatments. The data analysis was performed using R 3.0.3 statistical software. $P$ value levels $\leq 0.05$ were considered as statistically significant. We used generalized additive logistic model with natural cubic splines for predictive stone-free 
and postoperative fever models, which allow a more global comparison between two of the treatments. The best models were selected through generalized cross-validation (GCV) based on minimum UBRE scores.

\section{Results}

\section{Baseline Characteristics}

In our present study, we analyzed 62 medical records of patients treated by percutaneous lithotripsy versus 115 records involving retrograde intrarenal surgery. It is a retrospective, observational study, which may be subject to confounding. In this section, we compare the composition of the two treatment groups to see how likely confounding actually is. The basic characteristics of the patients records are shown in Table 1. In RIRS and PCNL, the mean age of the patients were 50.2 and 53.8 years respectively. Patients had single and multiple kidney stones of various sizes in both of the treatments. A total of 95 stones were present in the PCNL group and 179 stones in the RIRS group, respectively. In both of the treatments $61 \%$ of the patients had single stones and $39 \%$ of patients had multiple stones. The stone diameters in PCNL were higher. This final observation corresponds to the official guideline to use PCNL for larger stones and RIRS for smaller stones. Nevertheless, quite a wide variety of stone sizes were treated by both treatment regimes. Given the measured covariates, it does not look that confounding plays a particularly strong role in the analysis.
RIRS has Lower Postoperative Fever Rates than PCNL

Sepsis is one of the life-threatening postoperative complications associated with PCNL and RIRS. In this study, we compare the effect of several variables on the postoperative fever rates between the two interventions. There are some important factors in each of the treatments that significantly increase the risk of postoperative fever.

The factors that were found predictive for postoperative fever using a generalized additive logistic model in combination with AIC were age, gender, postoperative nephrostomy, preoperative urinary DJ stent, and operation time. We found that PCNL in general has higher rates of postoperative fever than RIRS. For both treatments, postoperative fever is less prevalent among older patients, among men, and among patients that do not have any urinary diversion. Figure 1a shows the risk of postoperative fever across age and gender of patients, when they do not have any type of urinary diversion and on an average 50-min operation time. It is clearly visible in the plot that for both of the treatments female patients have higher risk of postoperative fevers compared to males. The odds of women developing postoperative fevers after a PCNL intervention are 9.1 times higher than those of men. The odds of women developing postoperative fever after a RIRS intervention are 2.4 times higher than those of men. Particularly for PCNL long interventions negatively affect postoperative complications. Figure $1 \mathrm{~b}$ shows that for PCNL the odds of postoperative fevers of are increased by $6.3 \%$ with every additional minute in operation time. Although we found that post-PCNL fever
Table 1 Baseline stone and patient characteristics: PCNL versus RIRS

\begin{tabular}{lll}
\hline Stone burden & RIRS & PCNL \\
\hline Number of patients & 106 & 62 \\
Number of procedures & 115 & 62 \\
Age & $50.2 \pm 14.5$ & $53.8 \pm 11.7$ \\
Gender & & \\
Males & $65(57 \%)$ & $37(60 \%)$ \\
Females & $50(43 \%)$ & $25(40 \%)$ \\
Average number of renal calculi & $1.6 \pm 0.8$ & $1.5 \pm 0.7$ \\
Stone size (mm) & $17.3 \pm 7.3(5.9-47.7)$ & $28.6 \pm 12(13-76)$ \\
Stone side & & \\
Left kidney (sx) & $67(58 \%)$ & $27(44 \%)$ \\
Right kidney (dx) & $48(42 \%)$ & $35(56 \%)$ \\
Mean OR per patient (min) & $56.2 \pm 14.4(20-90)$ & $44.9 \pm 16.6(20-95)$ \\
Previous treatment ESWL & $13(11 \%)$ & $23(37.1 \%)$ \\
Hydronephrosis & $56(48.7 \%)$ & $17(27.4 \%)$ \\
Major complication (sepsis) rate & $17(14.8 \%)$ & $11(17.7 \%)$ \\
Minor complication rate & $7(6.1 \%)$ & $3(4.8 \%)$ \\
\hline
\end{tabular}



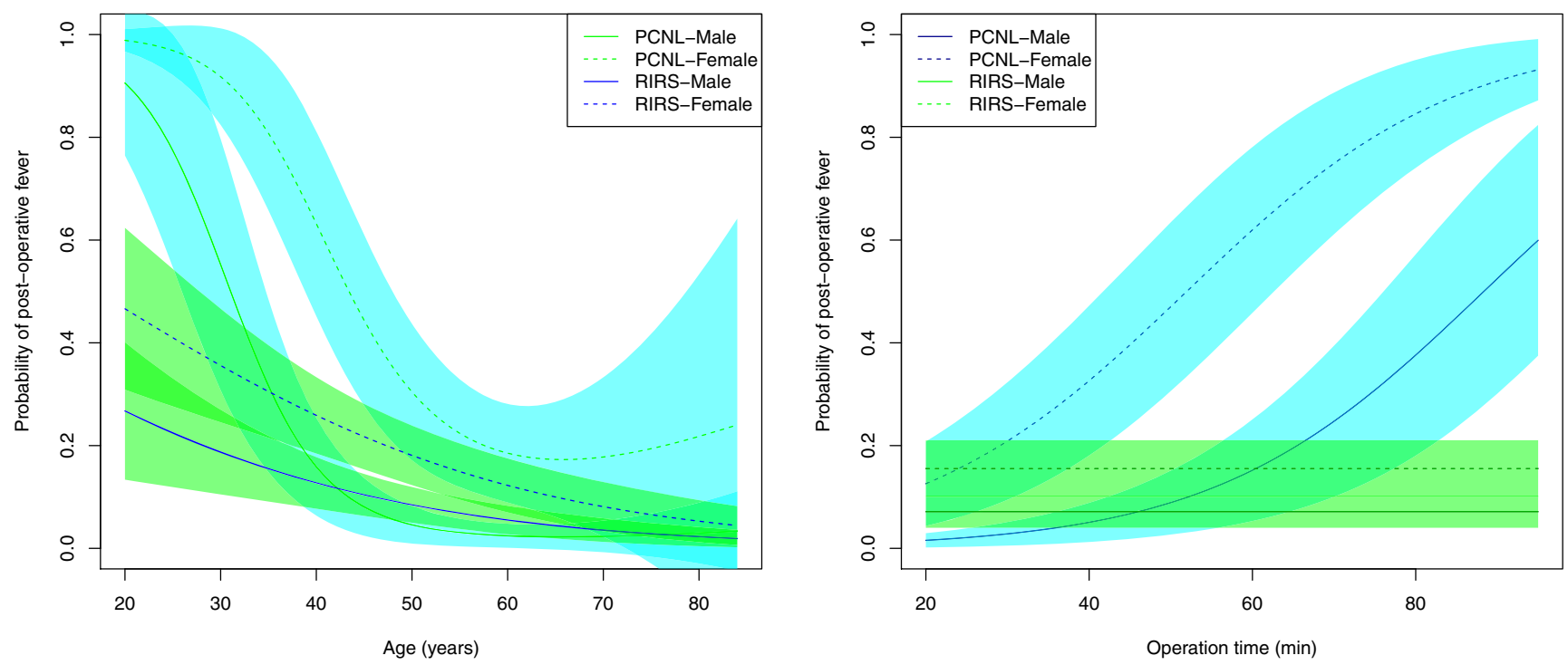

Fig. 1 Predictive risk of developing postoperative fever for PCNL versus RIRS (a) age (b) operation time (with standard errors)

risks are acceptably low, i.e. below $10 \%$, in male patients over 45, the overall risk of postoperative fevers is in general much lower for RIRS patients.

\section{Until 2-cm Diameter RIRS is Competitive in Effectiveness}

The effectiveness of retrograde intrarenal surgery and PCNL are found to depend significantly on the stone diameter, the density of stones and the stone location. Stone diameter is an important predictor and significantly influences stone-free rates in PCNL $(p=0.018)$ as well RIRS $(p=0.008)$. Both treatments are effected very similarly by an increase in stone diameter, reducing the odds of a successful intervention by slightly over $10 \%$ per $1 \mathrm{~cm}$ increased in diameter, keeping all other variables constant. Stone density seems to affect only the RIRS treatment, as can be seen in Fig. 2, resulting in poor results for somewhat brittle (350-650 HU) and very hard stones ( $>1100 \mathrm{HU})$. PCNL is negatively affected if stones are located in the middle calyx.

In Fig. 3, we compared PCNL and RIRS stone-free probabilities in terms of stone diameter for patients with one stone and average stone density of $982 \mathrm{HU}$. In general, it shows that removing a stone in the middle calyx by PCNL is as effective as removing it via RIRS. Whereas, it is clear that, overall, PCNL outperforms RIRS in terms of first-time effectiveness, measured as a stone-free status 3 months after the first intervention, up to 2-cm RIRS has a competitive treatment performance, as evidenced by an $81 \%$ stone-free rate for stones of $2 \mathrm{~cm}$ in diameter.

\section{Stone Density Influence on Operation Time}

The stone density is significantly associated with operation time both in percutaneous lithotripsy $(p=0.022)$ and retrograde intrarenal surgery ( $p=0.035$ ). In RIRS, as displayed in Fig. 4, operation time is stable around an average of 55 mins until $900 \mathrm{HU}$. After $900 \mathrm{HU}$, the average operation time increases to 63 mins. This is probably because it takes time during RIRS to pulverize hard stones. In PCNL, the

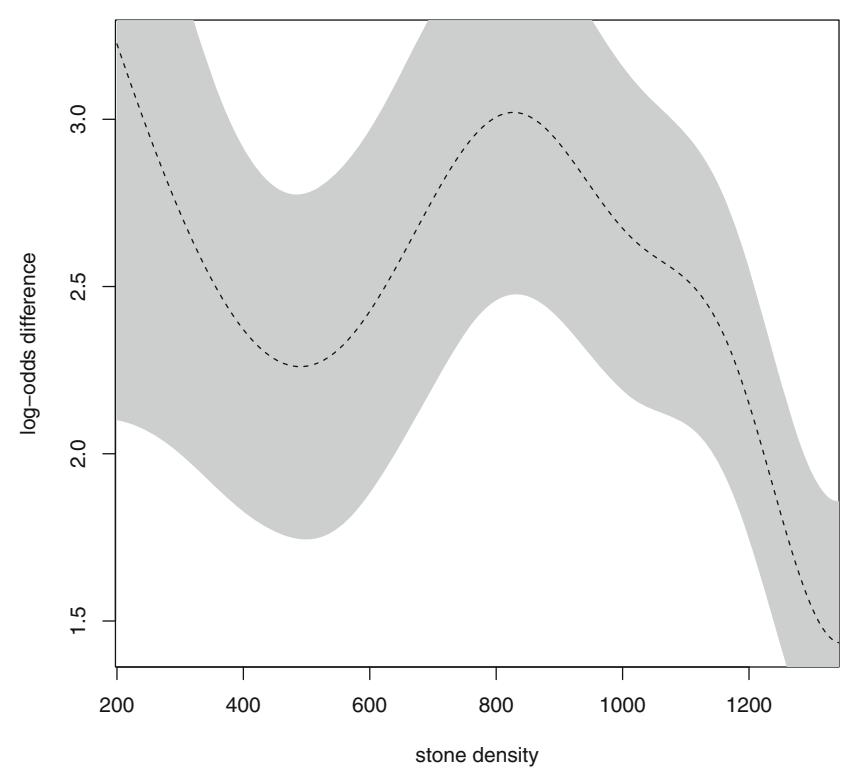

Fig. 2 Estimated curve of stone-free probability after 3 months on mean stone density (HU) with stantard errors 


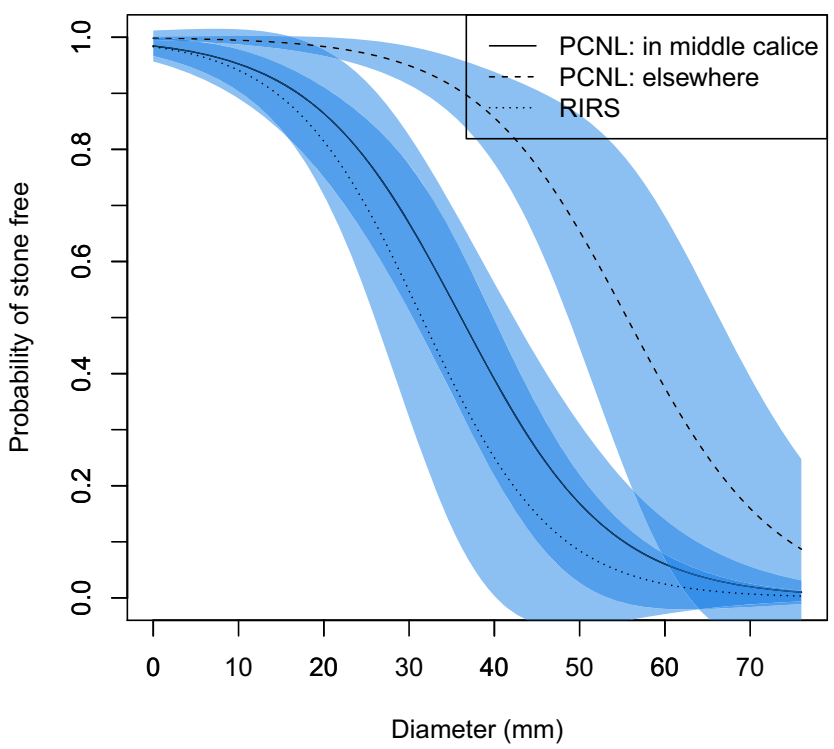

Fig. 3 Probability of treatment effectiveness of PCNL versus RIRS as a function of stone diameter and stone location (with standard errors)

density of the stone is negatively associated with operation time. For the stone density, $400 \mathrm{HU}$ operation time starts on average at 74 mins, which decreases constantly until 1000 HU stone density. As stones have to be removed and not pulverized, the data suggest that harder stones are easier and, therefore, faster to treat. After $1000 \mathrm{HU}$ stone density, the mean operation time levels out.

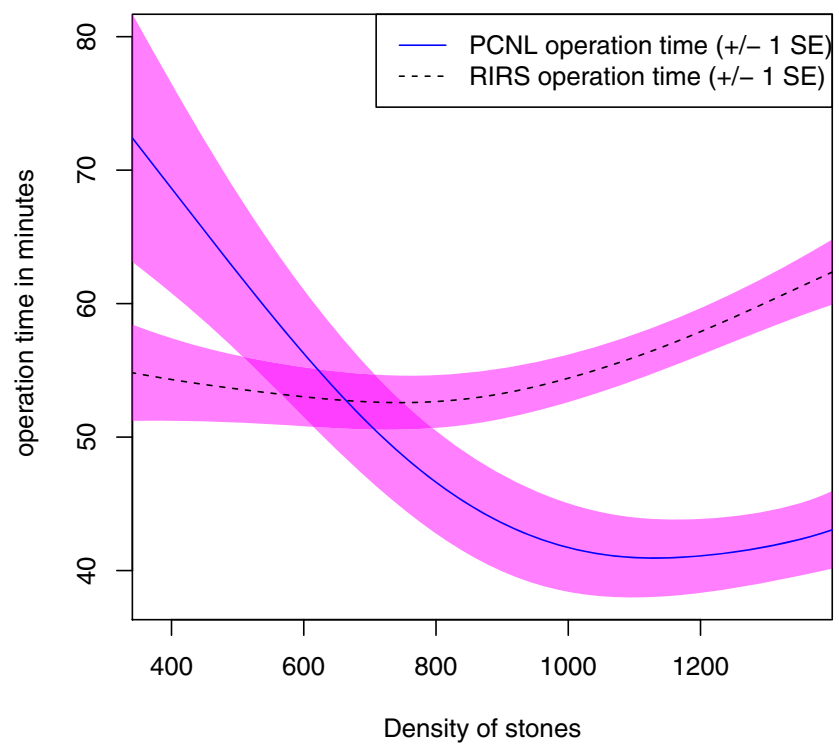

Fig. 4 Relationship between stone density and operation time for PCNL and RIRS

\section{Discussion}

The main drawback of the study is its retrospective nature (evidence level 2c) with a descriptive study design. The RIRS and PCNL treatments were not assigned randomly to the patients, but on the basis of the expert opinion of the urologists. This selection may induce compensatory effects. Knowing that PCNL is a very effective treatment, this may have been reserved for the most difficult cases, resulting in a deflated success rate or an increased postoperative fever rate. Therefore, we have analyzed the data to compensate for possible confounding. For the measured variables, with the exception of stone size, the two treatment groups are very similar, suggesting that there are no measured confounders. Clearly, the reported treatment effectiveness was corrected for stone size, whereas the postoperative fever rates were corrected for operation time, allowing for a fair comparison between RIRS and PCNL.

In our study, we have confirmed previous findings that PCNL has higher stone-free rates compared to RIRS. The stone diameter is an important predictor stone-free rates in both of the treatments, where larger stone diameter leads to lower treatment effectiveness. Each increase in diameter by $1 \mathrm{~cm}$ reduces the odds ratio of success by slightly over $10 \%$. It was found that PCNL has low effectiveness rates for stones in the middle calyx. It is hypothesized that the reason for the low stone-free rates of middle calyx is the fact that the majority of the PCNL punctures are made in the lower and upper calyx, from where it is difficult to reach stone locations in the middle calyx.

There is ample evidence that preoperative, positively tested urine culture is one of the important predictors of developing postoperative fever. In our study, all patients with urinary tract infection were treated 5 days prior to their intervention with specific antibiotics. In our current study, we analyzed the intraoperative and postoperative risk factors. We found that the patient's age is one of the most important predictor factor that significantly increases the risk of sepsis after either RIRS and PCNL treatment. Our finding shows that younger patients have a significant risk of developing sepsis. Gutierrez [5], in a recent study, concludes that along with preoperative positive urine culture, young age, preoperative nephrostomy, also diabetes, and staghorn stones were predictive factors for postoperative fever among PCNL patients.

Sepsis has been reported as one of important causes of mortality among patients particularly women after PCNL and ureteroscopy. A study reported that among 700 patients, who were treated with PCNL or an endoscopic procedure for upper UTI stones, 9 patients developed sepsis and all 
of them were female. Furthermore, 7 of them died [6]. A cross-sectional study based on 217 PCNL patients reported that gender was an important predictor associated with postPCNL fever risk, together with the use of nephrostomy tube and preoperative positive urine culture. They concluded that higher fever among females might be due to the propensity of urinary tract infection [1]. In our study, we found that postoperative fever rates for PCNL were much higher than those for RIRS.

\section{Conclusions}

We have found that PCNL has higher stone-free rates in comparison with RIRS. However, in terms of complications PCNL has very high postoperative fever rates, particularly for female patients and patients younger than 45 . We would recommend to use RIRS for stones up to $2 \mathrm{~cm}$ in diameter, due to its lower incidence of postoperative fever combined with its solid stone-free rates above $80 \%$. Therefore, RIRS can be recommended as a safe alternative in patients with the above described risk factors for postoperative fever. Moreover, if the operation time of PCNL is expected to last more than 40 mins, we would recommend using RIRS.

\section{Author Contributions}

- NP Gill: Data analysis, manuscript writing

- L D'Arrigo: Data collection, manuscript writing

- EC Wit: Data analysis, manuscript writing

Funding Information PhD scholar N.P.Gill was funded by a scholarship from University of Sindh, Jamshoro under faculty development program (NO.SU/PLAN/F.SCH /127) approved by the Higher Education Commission (HEC) of Pakistan.

\section{Compliance with Ethical Standards}

Conflict of Interest The authors declare that they have no conflict of interest.

Ethical Approval All procedures performed in studies involving human participants were in accordance with the ethical standards of the institutional and/or national research committee and with the 1964 Helsinki declaration and its later amendments or comparable ethical standards.
Informed Consent Informed consent was obtained from all individual participants included in the study.

Open Access This article is distributed under the terms of the Creative Commons Attribution 4.0 International License (http:// creativecommons.org/licenses/by/4.0/), which permits unrestricted use, distribution, and reproduction in any medium, provided you give appropriate credit to the original author(s) and the source, provide a link to the Creative Commons license, and indicate if changes were made.

\section{References}

1. Aghdas FS, Akhavizadegan $\mathrm{H}$, Aryanpoor A, Inanloo H, Karbakhsh M. Fever after percutaneous nephrolithotomy: contributing factors. Surg Infect. 2006;7(4):367-371.

2. Akman T, Binbay M, Ozgor F, Ugurlu M, Tekinarslan E, Kezer C, Aslan R, Muslumanoglu AY. Comparison of percutaneous nephrolithotomy and retrograde flexible nephrolithotripsy for the management of 2-4 cm stones: a matched-pair analysis. BJU Int. 2012;109(9):1384-1389.

3. Bozkurt OF, Resorlu B, Yildiz Y, Can CE, Unsal A. Retrograde intrarenal surgery versus percutaneous nephrolithotomy in the management of lower-pole renal stones with a diameter of 15 to $20 \mathrm{~mm}$. J Endourol. 2011;25(7):1131-1135.

4. Ghani KR, Wolf JS. What is the stone-free rate following flexible ureteroscopy for kidney stones?. Nat Rev Urol. 2015;12(5):281288.

5. Gutierrez J, Smith A, Geavlete P, Shah H, Kural AR, de Sio M, Sesmero JHA, Hoznek A, de la Rosette J, Group CPS, et al. Urinary tract infections and post-operative fever in percutaneous nephrolithotomy. World J Urol. 2013;31(5):1135-1140.

6. O'keeffe N, Mortimer A, Sambrook P, Rao P. Severe sepsis following percutaneous or endoscopic procedures for urinary tract stones. Br J Urol. 1993;72(3):277-283.

7. Sanguedolce F, Liatsikos E, Verze P, Hruby S, Breda A, Beatty $\mathrm{J}$, Knoll $\mathrm{T}$, et al. Use of flexible ureteroscopy in the clinical practice for the treatment of renal stones: results from a large european survey conducted by the eau young academic urologistsworking party on endourology and urolithiasis. Urolithiasis. 2014;42(4):329-334.

8. Turk C, Knoll T, Petrik A. and members of the European Association of Urology (EAU) guidelines office. Guidelines on urolithiasis. EAU Guidelines. 2014. 